\title{
Review
}

\section{Application of Terahertz Waves to Food Science}

\author{
Masaya KawASE ${ }^{*}$ \\ Nagahama Institute of Bio-science and Technology, 1266 Tamura-cho, Nagahama, Shiga 526-0829, Japan
}

Received April 10, 2012; Accepted May 8, 2012

Terahertz (THz) wave has a unique character that other range waves do not have, and its application is widely studied. Technology relating THz wave, such as generation, detection and application, remarkably develops in recent. Many studies about application of THz wave to food analysis have been performed. This review explains the basics of $\mathrm{THz}$ wave as well as application of $\mathrm{THz}$ wave to food analysis. Moreover, statistical method in treatment of THz spectrum is reported.

Keywords: Terahertz (THz) wave, THz time domain spectroscopy (THz-TDS), coherent THz spectroscopy, imaging, partial least squire (PLS)

\section{Introduction}

Terahertz (THz) waves are electromagnetic waves ranging from 0.3 to $10 \mathrm{THz}$ (wavelength from $1 \mathrm{~mm}$ to $30 \mu \mathrm{m}$ ), as shown in Fig. 1. THz waves are unique, having properties of both electrical and optical waves. Various applications of $\mathrm{THz}$ waves have been reported in many fields, including medical diagnosis, pharmaceutical analysis and security enhancement (Nagel et al., 2003; Woodward et al., 2003; Yamamoto et al., 2004a, 2004b; Kawase et al., 2003; Hineno et al., 1974; Taday et al., 2003; Walther et al., 2002). Absorption spectra in the $\mathrm{THz}$ range provide rich information for both intermolecular and intramoleculer interactions, including inter-ring interactions of disaccharides (Hineno et al., 1974). Absorption spectra in the THz range are very sensitive to differences in crystal structure (Walther et al., 2000), and have been applied to the study of drug polymorphs (Taday et al., 2003).

THz time domain spectroscopy (THz-TDS) is the most common $\mathrm{THz}$ spectroscopy method. $\mathrm{THz}$ pulses are mainly generated using femtosecond laser pulses, coherent transition radiation or free electron laser (FEL). The THz pulses generated by femtosecond laser pulses are useful for measuring the optical properties in wave number (frequency) ranges below $100 \mathrm{~cm}^{-1}(3 \mathrm{THz})$, and give a better signal-to-noise ratio than the far infrared. Fourier transform (FIR FT-IR) spectroscopy. (Grischkowsky et al., 1990) THz spectra are sensitive to the assembly state of molecules, which is considered to vary between optical isomers. Because of such characteristics of THz spectra, they could used to distinguish between the optical isomers.

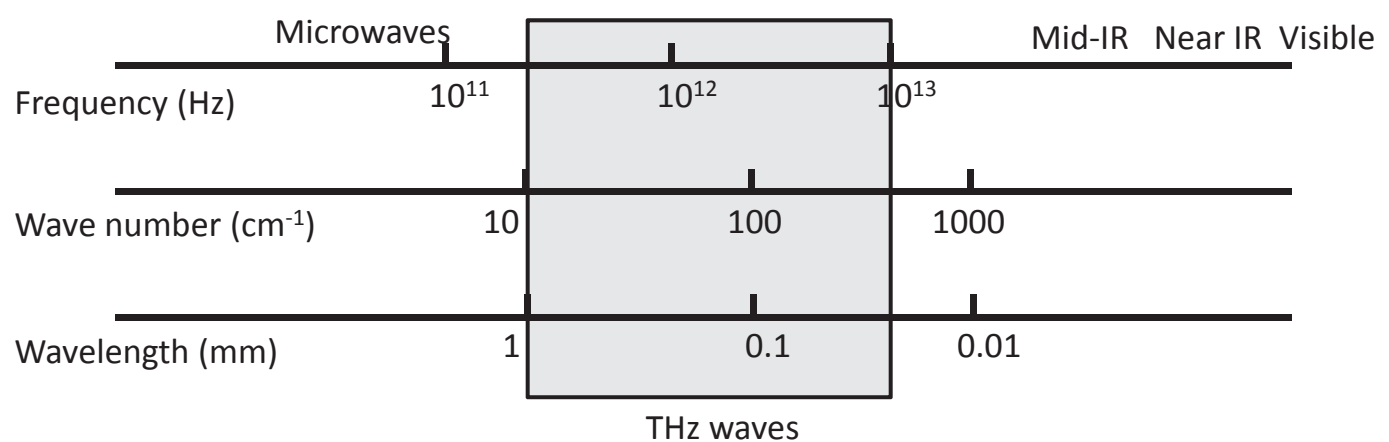

$3 \mathrm{THz}=3 \times 10^{12} \mathrm{~Hz}=100 \mathrm{~cm}^{-1}=0.1 \mathrm{~mm}$

*To whom correspondence should be addressed.

Fig. 1. $\mathrm{THz}$ waves.

E-mail: m_kawase@nagahama-i-bio.ac.jp 
$\mathrm{THz}$ waves generated by coherent transition radiation or FEL have the same properties as those generated by femtosecond laser pulses. Coherent $\mathrm{THz}$ waves are stronger than those generated with femtosecond laser pulses. (Takahashi et al., 1998; Takahashi 2005; Awano and Takahashi 2008), and have attracted attention in the recent development of FEL.

In this review, the basics of $\mathrm{THz}$ waves and their application will be reported, and the application of $\mathrm{THz}$ waves in food science is discussed.

\section{Generation and Detection of $\mathrm{THz}$ Waves}

Optical systems using $\mathrm{THz}$ waves generated by femtosecond laser pulse Generally, THz waves are generated by irradiation of semiconductor with femtosecond laser pulses. One example is shown in follows. A mode-locked Ti:sapphire laser, with a repetition rate at $82 \mathrm{MHz}$, a pulse with around $100 \mathrm{fs}$ and its center wavelength around $800 \mathrm{~nm}$, was used as the pump source. The emitter and detector were a dipole-type photoconductive antenna on a low-temperature grown GaAs (LT-GaAs) substrate. (Hangyo et al., 2005) The antennas were excited and triggered by laser pulses with an average power of $10 \mathrm{~mW}$. The bias voltage applied to the emitter photoconductive antenna was $20 \mathrm{~V}_{\mathrm{p}-\mathrm{p}}$ modulated at $53 \mathrm{kHz}$. The $\mathrm{THz}$ radiation from the emitter was collected and focused onto the sample using a parabolic mirror. The $\mathrm{THz}$ radiation transmitted through the sample was collected and focused with another parabolic mirror onto the photoconductive antenna (Hangy et al., 2002). Hyper-spherical Si substrate lenses were used for both the emitter and detector antennas to reduce the total reflection in the GaAs substrates (Tani et al., 1997) and increase the beam collection efficiency. The photoconductive current signal was detected with a lock-in amplifier and its time-domain signal was obtained by scanning the optical delay of the probe pulse. By Fourier transformation of the time-domain signal, the amplitude spectra were obtained. To reduce the absorption due to water vapor in the ambient air, the $\mathrm{THz}$ beam path was filled with dry air. A representative optical system using femtosecond lasers, as mentioned above, is illustrated in Fig. 2.

Optical system using coherent $\mathrm{THz}$ waves Coherent $\mathrm{THz}$ waves generated by a coherent transition radiation process are a useful $\mathrm{THz}$ wave source. Coherent transition radiation is generated by an accelerator, such as a linear accelerator (LINAC) or synchrotron storage ring.

One example is as follows. In this example, $\mathrm{L}$ band LINAC is used. The generation system for transition radiation is shown in Fig. 3. The electron beam passes through the aluminum foil, and the backward transition radiation is emitted. As the millimeter-wave region aluminum was regarded as a perfect conductor (Ehreneich et al., 1963), the intensity of backward radiation is equal to that of the forward radiation. Coherent $\mathrm{THz}$ waves can also be generated by FEL, a recently developed light source.

Radiation was detected using a composite-type liquid helium-cooled silicon bolometer (Infrared Laboratories Inc.) after a Martin-Puplett type interferometer and sample. The signal was amplified by a lock-in amplifier and its timedomain signal was obtained. By Fourier transformation of the time-domain signal, the amplitude spectra were obtained.

\section{THz Spectrum of Organic Compound}

In $\mathrm{THz}$ region, low-frequency vibration or rotation mode of the molecules can be detected as absorption. In the case of crystals, density near the Fermi-edge is observed. Therefore,

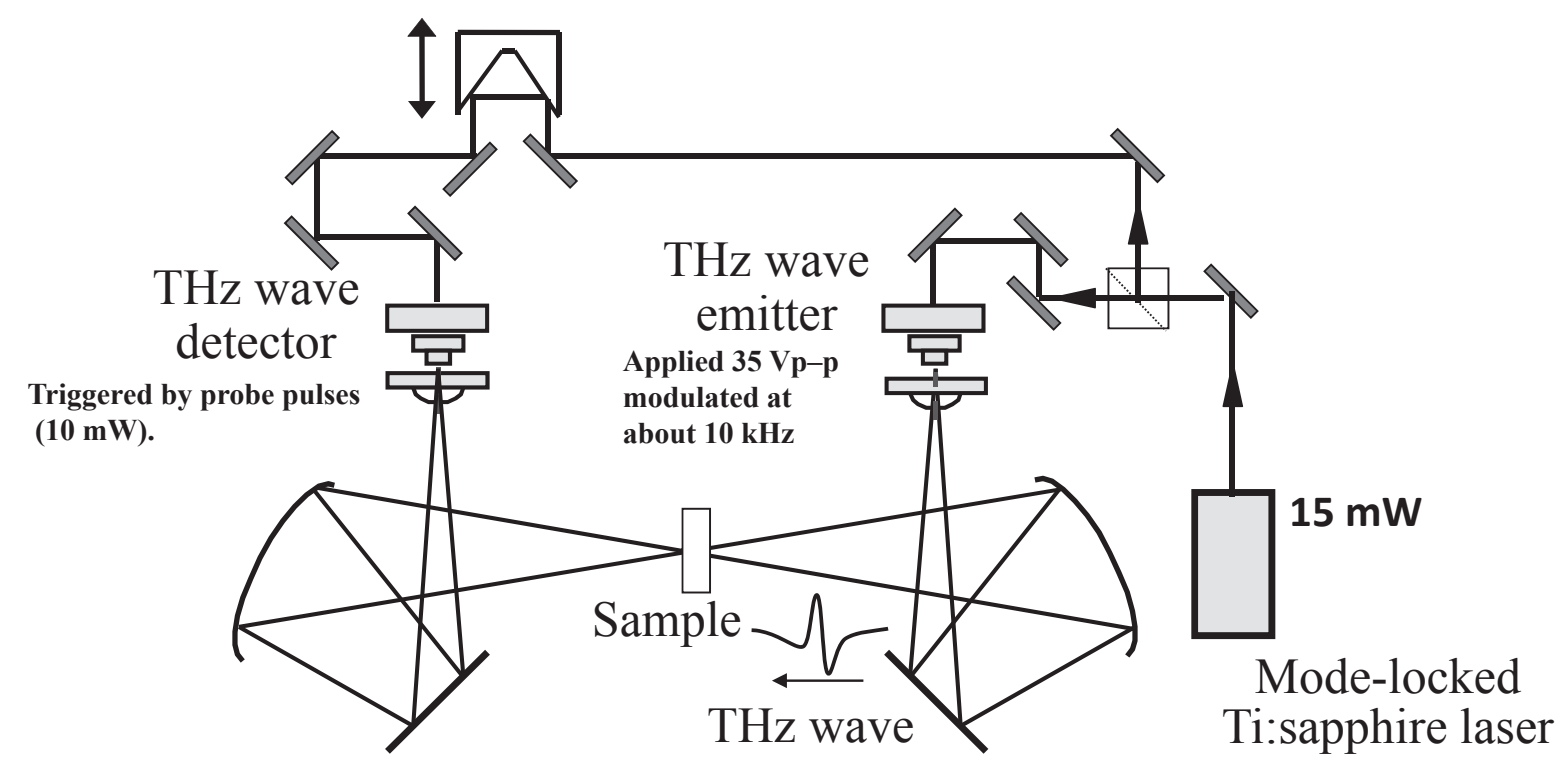

Fig. 2. Measurement system for $\mathrm{THz}$ absorption spectrum using femtosecond laser. 




Fig. 3. Measurement system for $\mathrm{THz}$ absorption spectrum using transition radiation.

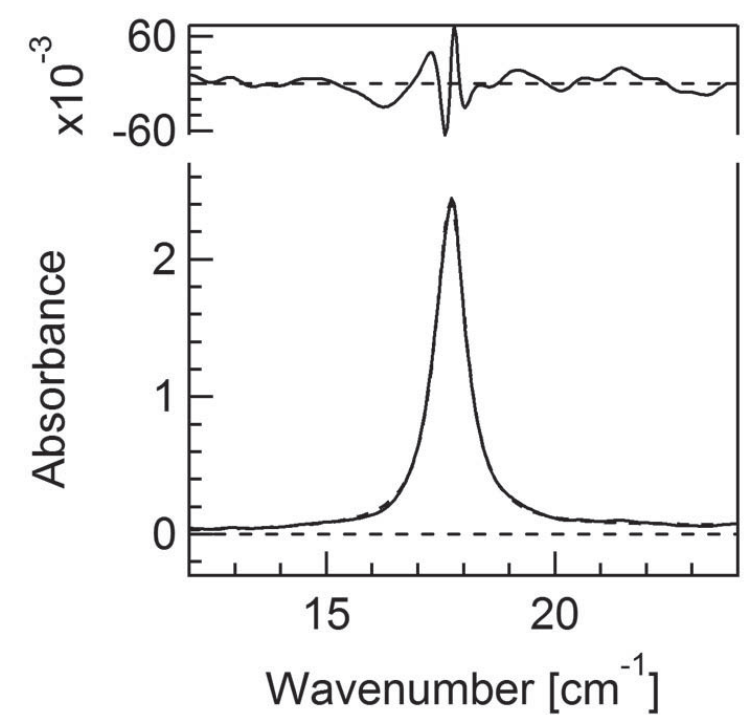

Fig. 4. THz-TDS spectrum of lactose.

the $\mathrm{THz}$ spectrum gives useful information on molecular or crystal structure, and this is different from the information obtained on mid- or near-infrared spectroscopy (Nshizawa, 2005).

In particular, THz-TDS spectroscopy gives the information about the dielectric properties of molecules or crystals (Nishizawa, 2005).

Recently, THz absorption measurement has been performed in many laboratories to obtain unique information by THz spectroscopy (Onishi et al., 2012).

In Figure 4, the THz-TDS spectrum for lactose is shown. The spectrum is very simple. Such simplicity is a powerful tool for recognition, identification and quantification. Application of such properties of $\mathrm{THz}$ waves has thus attracted attention in various fields.
Medicine and food are also active fields in which application of THz spectroscopy has been studied (Yamamoto et al. 2008).

\section{Application of THz-TDS in Drug Evaluation}

The recently developed femtosecond laser allows conventional use of THz-TDS in various fields. In security, THzTDS is able to detect drugs hidden in an envelope (Shibutani et al., 2005). This application demonstrates the usefulness of $\mathrm{THz}$ waves. THz-TDS has also been applied to drug management (Kawase, 2003).

Evaluation methods for drug safety are available are also applied to food. As noted in the Introduction, the THz spectrum is sensitive to the assembly state of molecules. This property is very useful for evaluating drug safety. Two typical examples are explained as follows.

The first is distinguishing original and generic medicines. Modasin, Mosyl and Mobenzocin, of which the active component is ceftazidime (Fig. 5), have been measured by THzTDS (Kawase et al., 2009). Figure 6 (A) shows the absorption spectra of Modacin, Mosyl and Mobenzocin. Modacin is the original medicine, while Mosyl and Mobenzocin are generic medicines. As shown in Fig. 6 (B), THz-TDS spectra differ between the original and generic medicines. The obtained results suggest that the THz-TDS method can effectively distinguish between original and generic medicines. Another is the example distinguishing between medicines before and after their expiration dates (Kawase et al., 2011). In such a study, it is necessary to measure the THz-TDS spectrum of the sample without destruction. In non-destructive measurement, the $\mathrm{THz}$ beam targets each sample at a 90 degrees angle of incidence, as shown in Fig. 6 (B), using an automatic sample folder, the Aispec model "mTR 100", with a $5 \mathrm{~mm}$ aperture diameter, as shown in Fig. 6 (A).

The obtained XRD pattern suggests that the polymorph of the effective compounds in Basen $\mathrm{OD}^{\mathrm{R}}(0.2 \mathrm{mg})$ remains

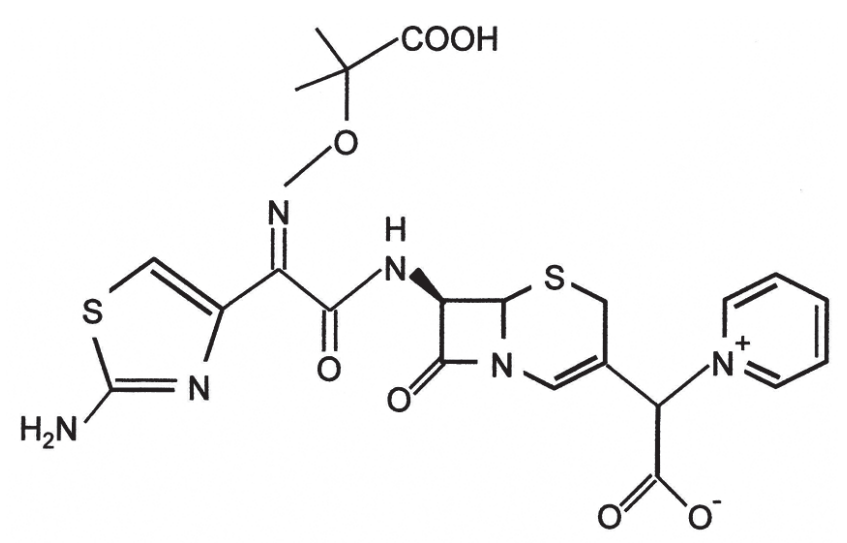

Fig. 5. Structure of ceftazidime. (Kawase et al., 2009). 



Fig. 6. The difference between the THz spectra of original and generic versions of ceftazidime Measured THz-TDS spectra is shown in A, and an enlarged spectra from 1.2 to $1.8 \mathrm{THz}$. Modacin is the original medicine, while Mosyl and Mobenzocin, are generic medicines. (Kawase et. al., 2009).
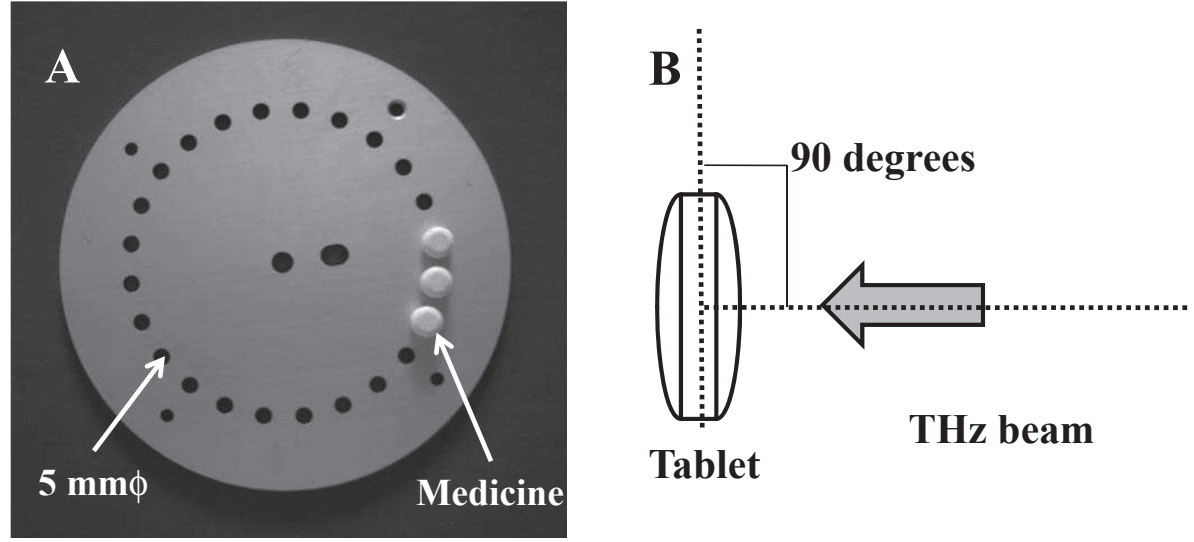

Fig. 7. Photograph of sample folder (a) and angle of incidence of $\mathrm{THz}$ beam to each sample (b). (Kawase et al., 2011).

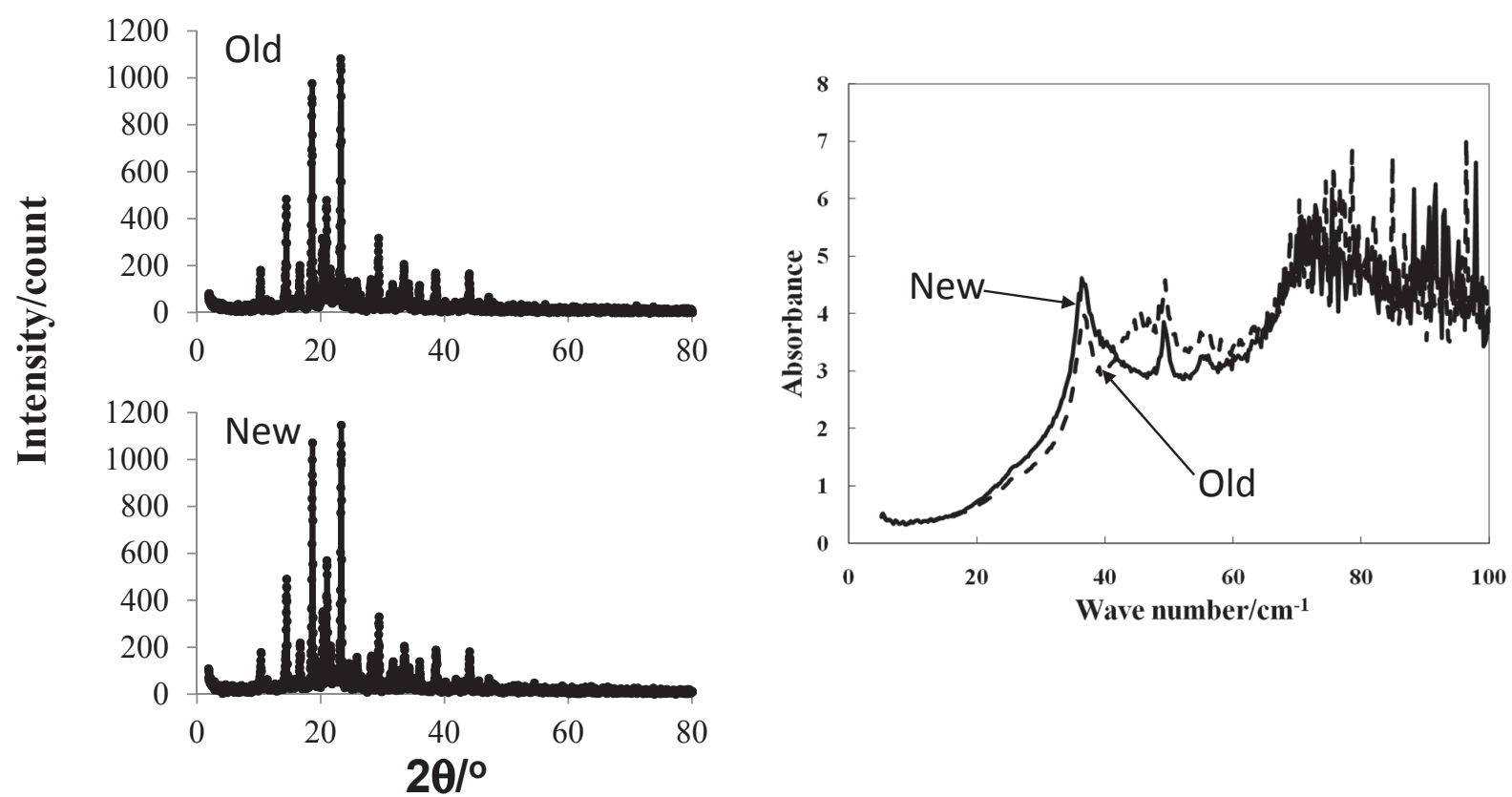

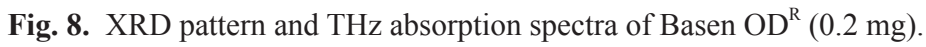

Bottom XRD and solid line in THz spectrum are those for new medicine, and upper XRD and dashed line in THz spectrum are those for old medicine. (Kawase et al., 2011). 
unchanged after the respective expiration dates, as compared to those before the expiration date (Fig. 7 (A)). In Fig. 7 (B), the solid line indicates the new medicine, and the dashed line indicates the old medicine. Differences in THz absorption spectra before and after expiration dates were observed. THz-TDS can sensitively detect changes in medicine over time. The same phenomenon was found in Amlodin $\mathrm{OD}^{\mathrm{R}}$ (5 $\mathrm{mg}$ ) and Gaster $\mathrm{D}^{\mathrm{R}}(10 \mathrm{mg})$.

THz-TDS has not been measured in a liquid sample to date. Recently, improvements in measurement methods have indicated the possibility of measuring liquid samples (Tani et al., 2011: Ogawa et al., 2009). If spectra for liquid samples can be obtained by THz-TDS, evaluation of injection and in- travenous feeding agents would be possible, and the usefulness of THz-TDS would increase in food science.

\section{Application of THz-TDS in Food Science}

Many applications of THz-TDS in food science are reported as shown in Fig. 9. (Nishizawa, 2005; Gowen et al., 2012). Quality control of food is a particularly important application for which $\mathrm{THz}$ waves have been used.

Water is closely related to food quality. As shown in Fig. 10, THz waves are sensitive to water and their transmission is scarcely affected by microstructures in the target because of long wavelength. Therefore, the state of water in food or other materials can be observed clearly using $\mathrm{THz}$ waves. By

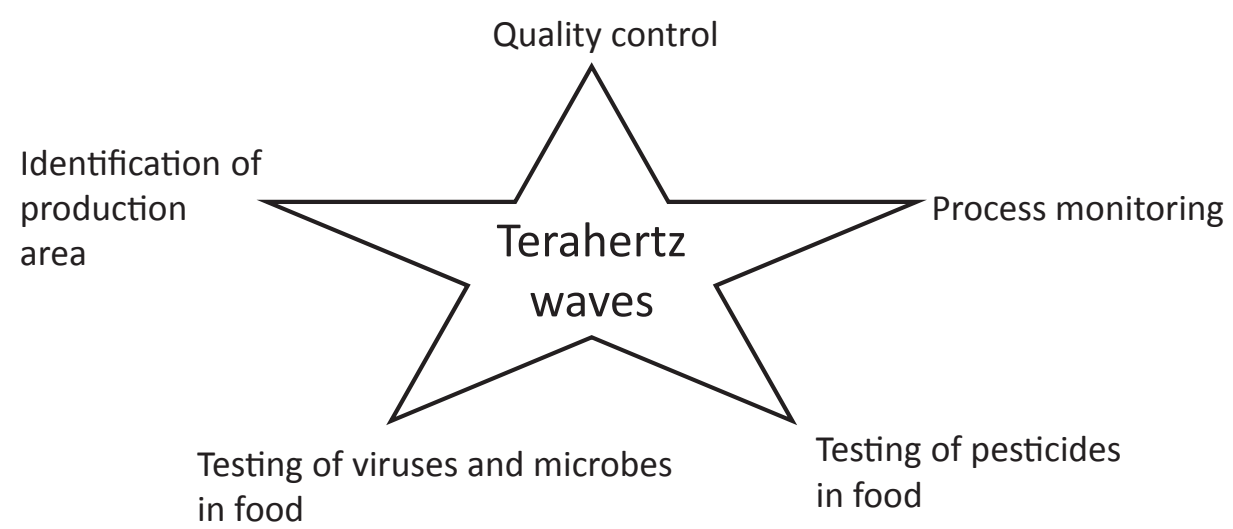

Fig. 9. Improved figure from Nishizawa (2005).

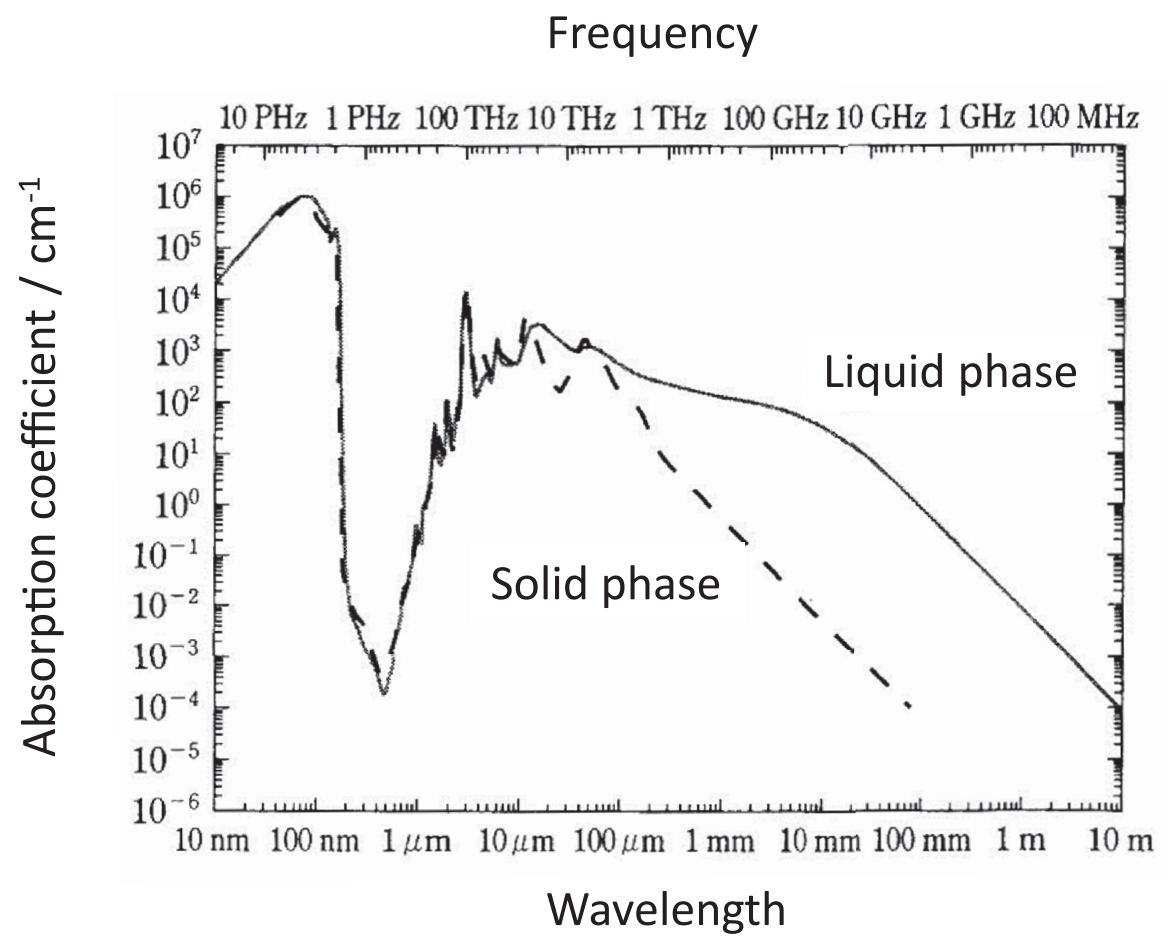

Fig. 10. Absorption coefficient of water in both solid and liquid phases. (Warren et al., 1984). 




Fig. 11. Transmission image of water in leaf. (Ogawa et al., 2004).

the $\mathrm{THz}$ imaging method, it is clear that water in the leaf decreases over time (Fig. 11). The internal structure of shrimp can be also observed clearly (Fig. 12).

Food safety is also an important application for $\mathrm{THz}$ waves. DNA, protein and polysaccharides have the absorption bands in the THz range (Park, 2012). THz waves can also selectively detect these materials. Therefore, $\mathrm{THz}$ waves can test for microbes, viruses and toxins, including prion, in food. As food is a heterogeneous material, imaging methods are employed for evaluation of food. An example of the selective imaging of chemicals is shown in Fig. 13. As each chemical has a different absorption wave form, selective

A

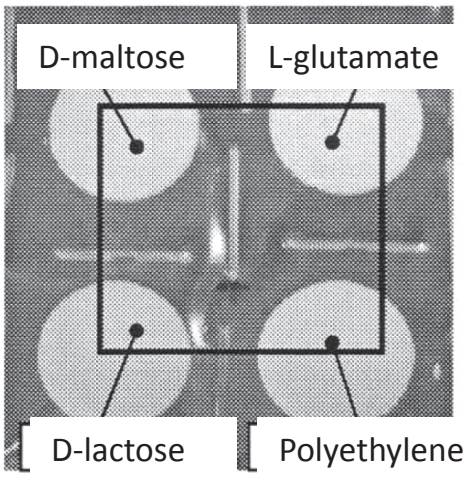

B



(c)

(d)

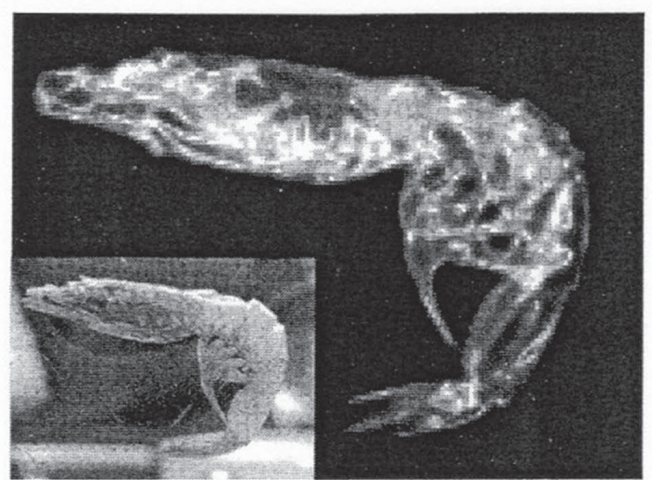

Fig. 12. Transmission image of shrimp by $\mathrm{THz}$ waves. (Kawase, 2004)

C

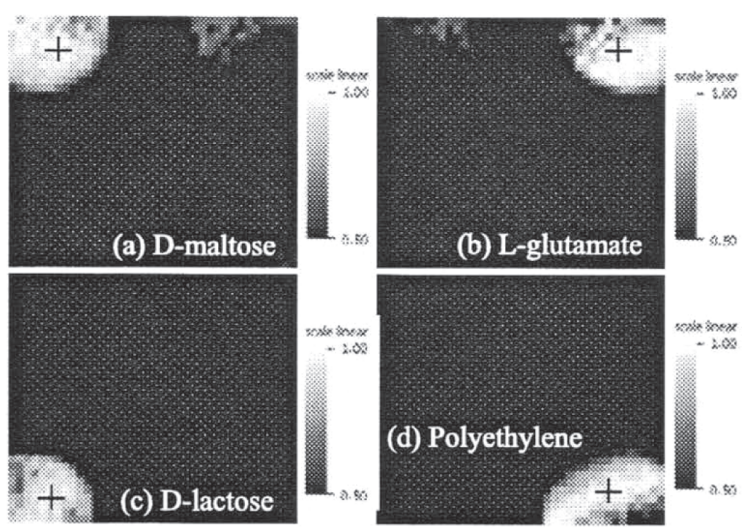

Fig. 13. Selective imaging of chemicals using THz waves.

A: Samples. B: Wave form of D-maltose (a), L-glutamate (b), D-lactose (c) and polyethylene (d). C: Image obtained from wave form in (B). (Onishi et al., 2012) 
images can be obtained. In addition, THz waves can be applied to evaluation of cheese quality (Sato et al., 2009), and the freshness of fruits, vegetables and eggs. Such studies are now being planned, the field will advance in the future.

\section{Quantification in THz Spectroscopy}

Yamaguchi et al. (2005) reported that absorption spectra in the $\mathrm{THz}$ range could distinguish DL- from L-alanine (or Dalanine), and suggested their usefulness in determination of the enantiometric composition of amino acids. The absorption intensity depends on the sample thickness, which varies slightly from sample to sample.

In general, THz-TDS absorption spectra of chemicals are very complex and often have very complex base lines. Therefore, a simple linear regression analysis using single peak intensity cannot precisely quantify the amount of chemicals. Therefore, improvements in quantitation have been attempted by multiple regression using some data points.

One example of quantification is shown for analysis of tartaric acid by THz-TDS (Nishokiori et al., 2008). Figure 14 shows the spectra of the absorption coefficient for L-, D- and DL-tartaric acid. The data points for quantification are indicated by arrows in Fig. 15. Multiple linear regression (MLR), partial least squares (PLS) (Hasegawa et al., 2000), and quadratic PLS (QPLS) (Hasegawa, et al., 1996) are employed for regression analysis. QPLS is a nonlinear regression method. The obtained decision coefficients were listed in Table 1 . These three methods gave a better decision coefficient. It has also been found that PLS and QPLS are better than MLR for quantitative analysis based on the THz-TDS absorption spectra.

In imaging, statistical methods are useful. Recently, Bayesian statistics have been applied to imaging methods



Fig. 14. Absorption Spectra of L-, D-, and DL-Tartaric Acids. Zero position of each spectrum is shown on vertical axis. Scale bar shows absorption coefficient of 5. (Nishikiori et al., 2008)



Fig. 15. Employed Absorption Bands for MLR, PLS and QPLS. (From Nishikiori et al., 2008)

Table 1. Summary of the employed multiple regression.

\begin{tabular}{c|c}
\hline Method & Decision coefficient \\
\hline MLR & 0.990 \\
PLS & 0.994 \\
QPLS & 0.995 \\
\hline
\end{tabular}

Multiple linear regression (MLR), partial least squares (PLS), and quadratic PLS (QPLS) were employed for regression analysis. QPLS is a nonlinear regression method. (From Nishikiori et al., 2008)

(Miyakoshi and Kato, 2012), resulting in clearer images and improved quality for evaluation.

As mentioned above, $\mathrm{THz}$ waves have unique characteristics not seen in any other frequency range. Studies using such characteristics are underway and it is hoped that $\mathrm{THz}$ waves will contribute to the development of food science.

\section{References}

Awano, T. and Takahashi, T. (2009). Coherent THz wave induced excitation in superionic conductors. J. Phys.: Conf. Ser., 148, 012040.

Ehrenreich, H., Philipp, H. R. and Segal, B. (1963). Optical properties of aluminuml. Phys. Rev., 132, 1918-1928.

Gowen, A. A., O’Sullivan, C. and O’Donnell, C.P. (2012). Terahertz time domain spectroscopy and imaging: emerging techniques for food process monitoring and quality control. Trends Food Sci. Technol., (available on line)

Grischkowsky D., Keiding S., Exter, M. and Fattinger, C. (1990). Far-infrared time-domain spectroscopy with terahertz beams of dielectrics and semiconductors. J. Opt. Sci. Am. B, 7, 2006-2015.

Hangyo, M., Nagashima, T. and Nashima, S. (2002). Generation and detection of terahertz pulsed radiation with photoconductive antennas and its application to imaging. Meas. Sci. Technol., 13, 
$1727-1738$.

Hangyo, M., Tani, M. and Nagashima, T. (2005). Terahertz timedomain spectroscopy of solids: A review. Int. J. Infrared Milli. Waves, 26, 1661-1690.

Hasegawa, K., Kimura, T., Miyashita, Y. and Funatsu, K. (1996). Nonlinear partial least squares modeling of phenyl alkylamines with the monoamine oxidase inhibitory activities, J. Chem. Info. Comput. Sci., 36, 1025-1029.

Hasegawa, K. and Funatsu, K. (2000). Partial least squares modeling and genetic algorithm optimization in quantitative structureactivity relationships, SAR and QSAR in Environmental Research, 11, 189-209.

Hineno, M. and Yoshinaga, H. (1974). Far-infrared spectra of mono-, di- and tri-saccharides in $50-16 \mathrm{~cm}^{-1}$ at liquid helium temperature. Spectrochim. Acta A, 30, 411-416.

Kawase, K., Ogawa, Y. and Watanabe, Y. (2003). Non-destructive terahertz imaging of illicit drugs using spectral fingerprints. Opt. Express, 11, 2549-2554.

Kawase, K. (2004). Terahertz imaging for drug detection and largescale integrated circuit inspection. Opt. Photonic. News., 15, 3439.

Kawase, M., Saito, T., Ogawa, M., Uejima, H., Hatsuda, Y., Kawanishi, S., Hirotani, Y., Myotoku, M., Ikeda, K., Takano, K., Hangyo, M., Yamamoto, K. and Tani, M. (2009). Terahertz absorption spectra of original and generics medicines of ceftazidime. Anal. Sci., 25, 1483-1485.

Kawase, M., Saito, T., Ogawa, M., Uejima, H., Hatsuda, Y., Kawanishi, S., Hirotani, Y., Myotoku, M., Ikeda, K., Konishi, H., Iga, I., Yamakawa, J., Nishizawa, S., Yamamoto, K. and Tani, M. (2011). Application of terahertz absorption spectroscopy to evaluation of aging variation of medicine. Anal. Sci., 27, 209-212.

Miyakoshi, Y. and Kato, S. (2012) Missing value imputation method by using bayesian network with weighted learning, IEEJ Trans. Electr. Inf. System., 132, 299-305.

Nagel, M., Richter, F., Haring-Bolivar, P. and Kurtz, H. (2003). Three-dimensional imaging of optically opaque materials using nonionizing terahertz radiation. Phys. Med. Biol., 48, 3625-3636.

Nishikiori, R., Yamaguchi, M., Takano, K., Enatsu, T., Tani, M., Silva, U.C., Kawashita, N., Takagi, T., Morimoto, S., Hangyo, M. and Kawase, M. (2008). Application of partial least square on quantitative analysis of L-, D-, and DL-tartaric acid by terahertz absorption spectra. Chem. Pharm. Bull., 56, 305-307.

Nishizawa, J. ed. (2005) "Teraherutsuha no kiso to ouyo" Kogyo Chosakai (in Japanese).

Ogawa, Y., Kawase, K., Mizuno, M., Yamashita, M. and Otani, C. (2004). Nondestructive and real-time measurement of moisture in plant. IEEJ Trans. Electr. Inf. System., 124, 1672-1677.

Ogawa, Y., Chang, L., Hayashi, S. and Fukunaga, K. (2009). Attenuated total reflection spectra of aqueous glycine in the terahertz region. IEICE Electro. Exp., 6, 117-121.
Onishi, Y., Akagi, M., Kitagishi, K. and Izutani, Y. (2012). THz spectroscopic imaging processing for extraction multiple samples. The $4^{\text {th }}$ International Workshop on Far-infrared Technologies 2012. 57.

Park, G. S., Ahn, C. B., Ahn, J., Choi, H., Han, H., Han, J. K., Jeong, Y. U., Kwon, O. S., Kim, K., Kim, Y., Park, I., Park, J., Park, W. Y., Son, J. H., Song, Y. K., Kim, P. and Choi, H. (2012). Research on biomedical systems using THz radiation. The $4^{\text {th }}$ International Workshop on Far-infrared Technologies 2012. 31.

Sato, H., Ogawa, Y. and Watanabe, K. (2009). Feasibility of the evaluation of cheeses using terahertz spectroscopy. J. Illum. Engng. Inst. Jpn., 93, 481-486. (in Japanese)

Shibuya, T., Yamashita, M., Ogawa, Y., Otani, C., Kawase, K., Inoue, H. and Kanamori, T. (2005). Nondestructive Inspection by Using Scattering and Spectra in Terahertz Waves. IEEJ Trans. Electr. Inf. System., 125, 545-550.

Takahashi, T., Matsuyama, T., Kobayashi, K. and Fujita, Y. (1998). Utilization of coherent transition radiation from a linear accelerator as a source of millimeter-wave spectroscopy. Rev. Sci. Instr. 69, 3770-3775.

Takahashi, T. (2005) Coherent synchrotron radiation. Kasokuki, 2, 11-19 (in Japanese).

Taday, P. F., Bradley, I. V., Arnone, D. D. and Pepper, M. (2003). Using terahertz pulse spectroscopy to study the crystalline structure of a drug: A case study of the polymorphs of ranitidine hydrochloride. J. Pharm. Sci., 92, 831-838.

Tani, M., Matsuura, S. and Sakai, K. (1997). Emission characteristics of photoconductive antennas based on low- temperaturegrown GaAs and semi-insulating GaAs. Appl. Opt., 36, 78537859.

Tani, M., Horita, K., Kinoshita, T., Que, C. T., Estacio, E., Yamamoto, K. and Bakunov, M. I. (2011). Efficient electro-optic sampling detection of terahertz radiation via Cherenkov phase matching. Optics Express, 19, 19901-19906.

Yamaguchi, M., Miyamaru, F., Yamamoto, K., Tani, M. and Hangyo, M. (2005). Terahertz absorption spectra of L-, D-, and DL-alanine and their application to determination of enantiometric composition. Appl. Phys. Lett., 86, 53903.

Yamamoto, K., Yamaguchi, M., Miyamaru, F., Tani, M., Hangyo, M., Ikeda, T., Matsushita, A., Koide, K., Tatsuno, M. and Miyami, Y. (2004a). Noninvasive inspection of C-4 explosive in mails by terahertz time-domain spectroscopy. Jpn. J. Appl. Phys., 43, L414-L417.

Yamamoto, K., Yamaguchi, M., Tani, M., Hangyo, M., Teramaru, S., Isu, T. and Tomira, N. (2004b). Degradation diagnosis of ultrahighmolecular weight polyethylene with terahertz-time-domain spectroscopy. Appl. Phys. Lett., 85, 5194-5196.

Yamamoto, K., Tani, M. and Hangyo, M. (2008). Introduction of terahertz time-domain spectroscopy and its application to molecular science studies. Ekisho, 12, 94-110. (in Japanese). 
Walther, M., Plochocka, P., Fisher, B., Helm, H. and Uhd Jepsen, P. (2000). Far-infrared vibrational spectra of all-trans, 9-cis and 13cis retinal measured by $\mathrm{THz}$ time- domain spectroscopy. Chem. Phys. Lett., 332, 389-395.

Walther, M., Plochocka, P., Fisher, B., Helm, H. and Uhd Jepsen, P. (2002). Collective vibrational modes in biological molecules investigated by terahertz time-domain spectroscopy. Biopolymers,
67, 310-313.

Warren, S. G. (1984). Optical constants of ice from the ultraviolet to the microwave. Appl. Opt. 23, 1206-1225.

Woodward, R. M., Wallace, V. P., Pye, R. J., Cole, B. E., Amone, D. D., Linfield, E. H. and Pepper, M. (2003). Terahertz pulse imaging of ex vivo basal cell carcinoma. J. Invest. Dermatol., 120, 7278. 